\title{
Risk factors for cage retropulsion after transforaminal lumbar interbody fusion in older patients
}

\author{
Nan $\mathrm{Li}^{1 \#}$, Min Dai ${ }^{2 \#}$, Bin Zhang ${ }^{2 \#}$, Da He ${ }^{1}$, Yi Wei ${ }^{1}$, Fangfang Duan ${ }^{1}$, Yuqing Sun ${ }^{1}$, Bo Liu ${ }^{1}$, Fengbo Mo ${ }^{2}$, \\ Wei Tian ${ }^{1}$ \\ ${ }^{1}$ Department of Spine Surgery, Beijing Jishuitan Hospital, The Fourth Medical College of Peking University, Beijing, China; ${ }^{2}$ Department of \\ Orthopaedics, The First Affiliated Hospital of Nanchang University, Nanchang, China \\ Contributions: (I) Conception and design: W Tian, N Li, M Dai; (II) Administrative support: Beijing Jishuitan Hospital; (III) Provision of study \\ materials or patients: N Li; (IV) Collection and assembly of data: B Zhang, D He, F Mo; (V) Data analysis and interpretation: Y Wei, F Duan, Y Sun; \\ (VI) Manuscript writing: All authors; (VII) Final approval of manuscript: All authors. \\ \#These authors contributed equally to this work. \\ Correspondence to: Wei Tian, MD, PhD, Professor. Department of Spine Surgery, Beijing Jishuitan Hospital, The Fourth Medical College of Peking \\ University, 31 Xinjiekou St., Xicheng District, Beijing, China. Email: drtianweia@163.com.
}

\begin{abstract}
Background: To investigate the incidence of cage retropulsion (CR) following transforaminal lumbar interbody fusion (TLIF) and the associated risk factors in older patients with lumbar disorders.

Methods: Between January 2015 and December 2017, 1,880 older patients (aged >60 years) who underwent open TLIF were preliminarily enrolled in this retrospective study. The patients' medical records were reviewed, and the risk factors potentially associated with CR were analyzed.

Results: A total of 1,662 patients (692 males and 970 females, with an average age of $68.7 \pm 5.2$ years) who met the eligibility criteria were finally enrolled in this study. Following TLIF, 29 older patients (1.74\%) developed CR including 12 patients with spinal stenosis, 7 patients with degenerative spondylolisthesis, 5 patients had degenerative disc diseases, 3 patients had surgical history, and 2 patients suffered isthmic spondylolisthesis. Of the 29 patients, 21 patients suffered lower back pain and/or sciatica (72.4\%), while 8 patients were asymptomatic (27.6\%). In multivariate analysis, screw loosening [odds ratio $(\mathrm{OR})=7.315 ; 95 \%$ confidence interval (CI): 3.4-15.7] and endplate injury (OR =4.947; 95\% CI: 2.3-10.6) were found to be independently associated with CR in older patients after TLIF.

Conclusions: The incidence of CR following TLIF in older patients is $1.74 \%$. Screw loosening and endplate injury are risk factors for CR in older patients with TLIF.
\end{abstract}

Keywords: Cage retropulsion (CR); lumbar disorders; older patients; risk factors; transforaminal lumbar interbody fusion (TLIF)

Submitted Sep 03, 2020. Accepted for publication Dec 10, 2020.

doi: $10.21037 /$ atm-20-7416

View this article at: http://dx.doi.org/10.21037/atm-20-7416

\section{Introduction}

Transforaminal lumbar interbody fusion (TLIF) was first developed by Harms and Rolinger in the early 1980s as a modification of posterior lumbar interbody fusion (1). TLIF has since been widely used to treat degenerative lumbar diseases. When achieving intervertebral fusion by a cage with auto-cancellous bone or allo-bone, it also provides anterior column support, restores the disc height, and decompresses the nerve root through reconstruction of the neuroforaminal height (2-4).

Despite the benefits of TLIF, however, a couple of complications have been reported in relation to the cage used in the procedure; one such complication is cage retropulsion (CR) (5). Several elements have been reported to be possible risk factors for $\mathrm{CR}$, such as bilateral 
facetectomy, multi-level fixation, fusion involves $\mathrm{S} 1$, an undersized cage, and a cage malpositioning. The incidence of CR is reported to be about $1 \%(0.8-1.3 \%)$, which is low and should be attributed to posterior fixation (6-9). Because strong fixation contributes to cage fusion with vertebrae. Therefore, any factors that compromise the strength of cage fixation, such as screw loosening caused by osteoporosis, could-at least theoretically-result in CR. However, as bone mineral density (BMD) tests are not a conventional preoperative examination for patients undergoing TLIF, especially in elderly patients, a conclusion on whether osteoporosis and CR are related has not been reached.

Hence, the present study aimed to investigate the incidence of CR in older patients undergoing TLIF, and to explore the risk factors for CR following TLIF in elderly patients.

We present the following article in accordance with the STROBE reporting checklist (available at http://dx.doi. org/10.21037/atm-20-7416).

\section{Methods}

\section{Patient enrollment}

This retrospective study was approved by the Institutional Review Board of Beijing Jishuitan Hospital (202007-24). All procedures performed in this study involving human participants were in accordance with the Declaration of Helsinki (as revised in 2013). Individual consent for this retrospective analysis was waived.

Between January 2015 and December 2017, 1,880 patients who underwent TLIF for lumbar degenerative disorders were enrolled and their clinical outcomes were analyzed. The inclusion criteria were: (I) patients aged $\geq 60$ years; and (II) a diagnosis of: spinal canal or foraminal stenosis, degenerative spondylolisthesis, isthmic spondylolisthesis, recurrent disc herniation or stenosis after previous surgery, or degenerative disc disease. The exclusion criteria were: (I) patients with spinal scoliosis; (II) patients who underwent oblique interbody fusion or a minimally invasive TLIF procedure; (III) patients who suffered from deep surgical site infection postoperatively. All patients underwent preoperative quantitative computed tomography (QCT) for the measurement of BMD. The criterion used to diagnose osteoporosis was a QCT value of $<80 \mathrm{mg} / \mathrm{cm}^{3}$, which is equivalent to a dual-energy $\mathrm{X}$-ray absorptiometry (DXA) T-score of -2.5 for the diagnosis of osteoporosis (10).

\section{Surgical technique and equipment}

The consistent TLIF procedures were performed by our experienced surgeons. A midline incision was made to expose the posterior elements and lateral aspects of the facets on both sides. Following bilateral pedicle screw fixation, ipsilateral laminotomy and facetectomy were performed to expose the intervertebral disc. Lateral recess and nerve root canal decompression were also carried out. The single cage was routinely inserted on the side with major symptoms. If the symptoms were equal on both sides, bilateral facetectomy and cage insertion were deemed necessary. After meticulous discectomy and thorough endplate preparation, the autologous bone graft from resected bone was milled and packed into the disc space, then, the fusion cage packed with milled bone was inserted into the disc space. To increase the local stability, appropriate compression was applied through the pedicle screws and rods. Finally, a C-arm was employed to confirm that all implants were positioned correctly. The cages used in this study were CONTACTTM Fusion Cage (titanium, Depuy Synthes, Raynham, MA, USA), Concorde (carbon fiber, Depuy Orthopaedics Inc., Warsaw, IN, USA), and Capstone (PEEK, Medtronic Sofamor Danek, Memphis, TN, USA).

\section{Radiological assessment}

CR was defined as the movement of the posterior margin of the cage into the spinal canal behind the posterior margin of the vertebral body (5). Screw loosening was defined as pullout of a pedicle screw on lateral radiography or a radiolucent area around the screws detected by computer tomography (CT) at the follow-up. According to the definition from a previous study (5), a pear-shaped disc was defined as a disc with a convex surface in the posterior halves of the superior and inferior endplates with a concave surface in the anterior halves. An endplate injury was defined as the cage breaching into the cortical endplate on the $\mathrm{X}$-ray immediately after surgery (9). Information regarding the type and height of the cages were documented from patients' medical records. The disc height was defined as the distance between the midpoints of the superior and inferior endplates from the preoperative sagittal image reconstruction of CT scan. An X-ray was conducted within 1 week after surgery, and at 3, 6, 12, and 24 months after surgery, respectively. If the $\mathrm{X}$-ray showed suspicious screw loosening, a CT scan was performed. 
Table 1 Patients' demographics

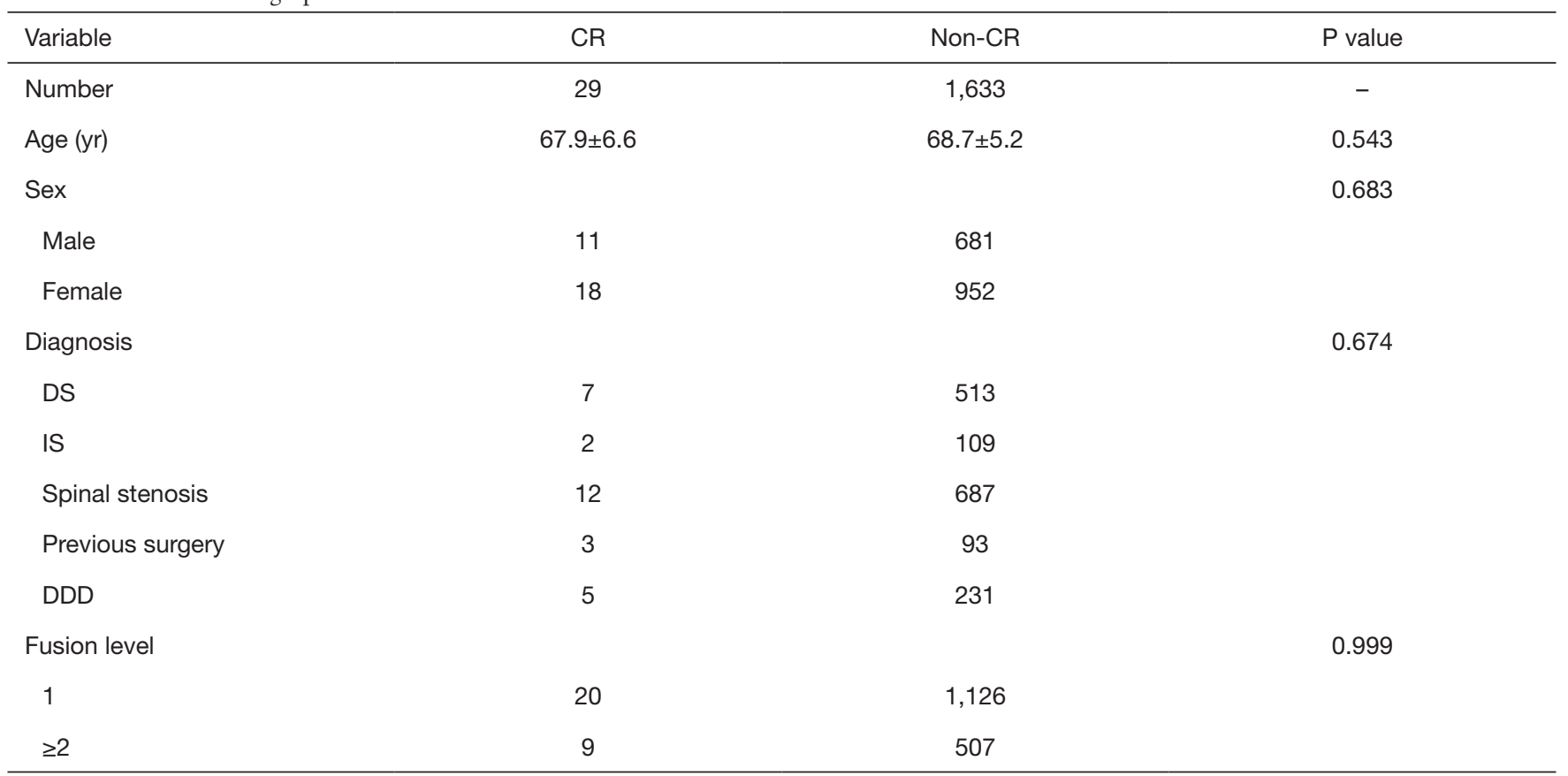

Values are presented as mean \pm standard deviation or number. CR, cage retropulsion; DS, degenerative spondylolisthesis; IS, isthmic spondylolisthesis; DDD, degenerative disc disease.

\section{Statistical analysis}

Statistical analyses were performed with SPSS 22.0 (IBM Corp., Armonk, NY, USA). The Shapiro-Wilk test was used to assess the normality of age and cage-disc height data. Data with normal distribution were described as the mean \pm standard deviation, while non-normally distributed data were described as the median (inter-quartile range, IQR). Differences in age and cage-disc height were compared between two groups using the independent samples $t$-test and Mann-Whitney $U$ test, respectively. Categorical variables were described as numbers and percentages, and were compared between groups using Pearson's Chisquared or Fisher's exact test. For the multivariate analysis, binary logistic regression was conducted using variables with statistical significance in the univariate analysis as independent variables. A $\mathrm{P}$ value $<0.05$ was considered to be statistically significant.

\section{Results}

Of the 1,880 older patients who underwent open TLIF, 218 patients were excluded due to unavailable medical records, incomplete data, or loss to follow-up. Finally, 1,662 patients (692 males and 970 females) were included in this study. These patients had an average follow-up of $26.1 \pm 8.8$ months (range, 12-43 months). After surgery, CR occurred in 29 patients (11 males and 18 females), with a postoperative incidence of $1.74 \%$. The mean time of CR occurrence was $3.1 \pm 2.1$ months after surgery (range, 4 days -8 months) . Of the 29 patients who developed CR, 12 had spinal stenosis, 7 had degenerative spondylolisthesis, 5 suffered degenerative disc disease, 3 suffered a surgical history, and 2 encountered isthmic spondylolisthesis. Twenty of these patients underwent single-segment spinal fusion, and the remaining 9 received fusion at 2 levels or more. There were no significant differences in age, sex, diagnosis, or fusion level between the CR and non-CR groups $(\mathrm{P}>0.05)$. The patients' characteristics are summarized in Table 1 .

\section{Clinical and radiological outcomes}

Among the 29 older patients with CR, 21 patients reported lower back pain and/or sciatica (72.4\%), and 8 patients were asymptomatic $(27.6 \%)$. Of the 21 patients with symptoms, 7 showed a good response to conservative treatments, while 
Table 2 The radiologic and surgical outcomes in CR and non-CR group

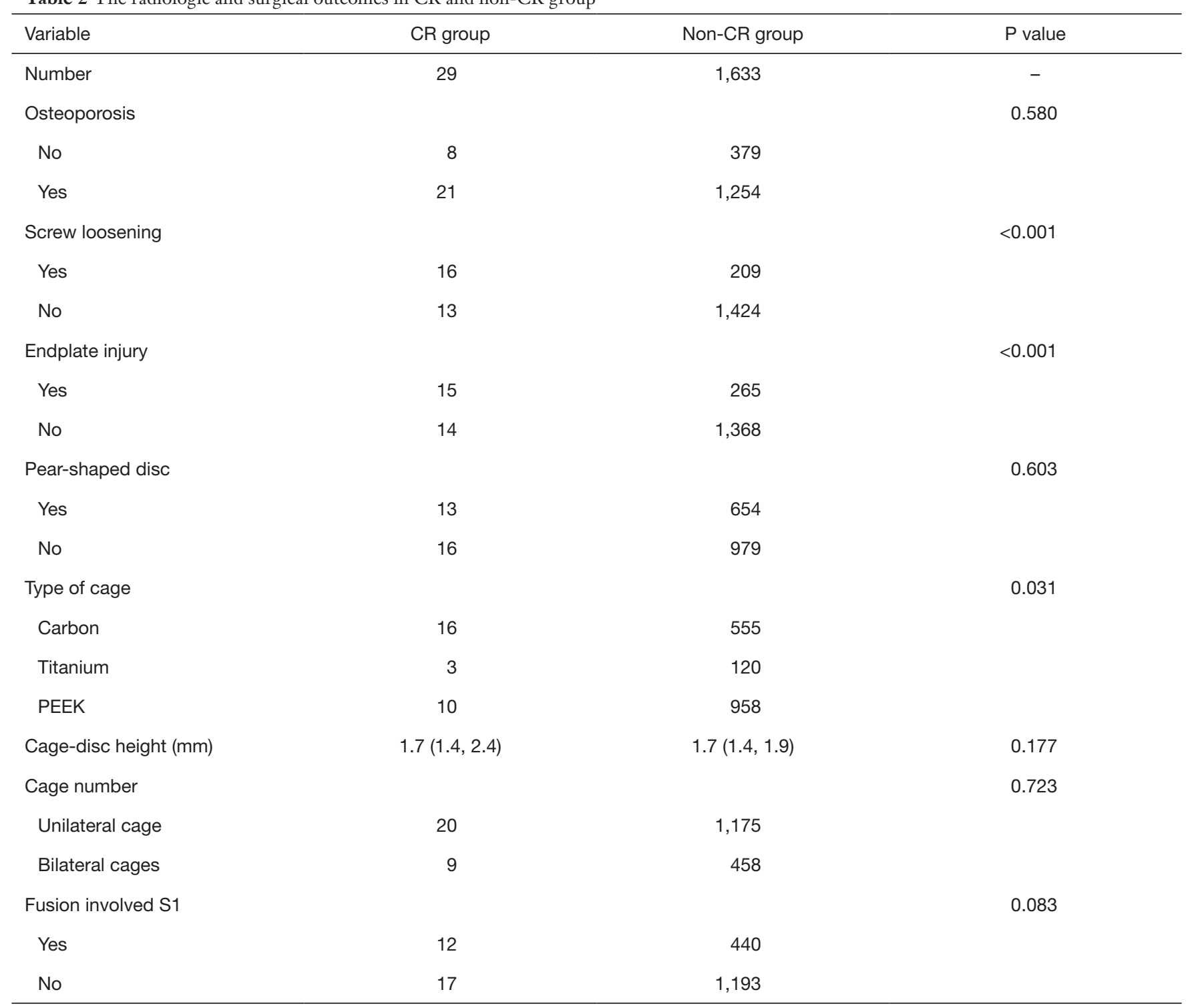

The value of cage-disc height was described as median (inter-quartile range, IQR). CR, cage retropulsion; PEEK, Poly-ether-ether-ketone.

the other 14 patients had to undergo revision surgery due to intractable pain and/or radiculopathy. Osteoporosis was diagnosed in $72.4 \%$ patients $(21 / 29)$, while screw loosening and endplate injury were observed in 16 and 15 patients, respectively. Significant differences were found in the distribution of screw loosening $\left(\chi^{2}=43.709, \mathrm{P}<0.001\right)$, endplate injury $\left(\chi^{2}=25.628, \mathrm{P}<0.001\right)$, and cage type $\left(\chi^{2}=6.929, \mathrm{P}=0.031\right)$ between the CR group and the nonCR group. However, no significant difference was observed in the incidences of osteoporosis, pear-shaped disc, cagedisc height, cage number, or fusion level between the two groups (all $\mathrm{P}>0.05$ ) (Table 2).

\section{Multivariate analysis of potential risk factors for $C R$}

The potential risk factors for CR after TLIF were analyzed in the 29 patients in the CR group. The univariate analysis showed that screw loosening, endplate injury, and cage type were associated with CR. However, in multivariate analysis, only screw loosening [odds ratio (OR): 7.315; 95\% confidence interval $(\mathrm{CI}): 3.412-15.684 ; \mathrm{P}<0.001$ ] and endplate injury (OR: 4.947; 95\% CI: 2.312-10.584; 
Table 3 Multivariate analysis on the cage retropulsion

\begin{tabular}{lcc}
\hline Variable & OR $(95 \% \mathrm{Cl})$ & $\mathrm{P}$ value \\
\hline Screw loosening & $7.315(3.412,15.684)$ & $<0.001$ \\
Endplate injury & $4.947(2.312,10.584)$ & $<0.001$ \\
Type of cage & & 0.138 \\
Titanium & $0.785(0.214,2.879)$ & 0.716 \\
PEEK & $0.436(0.192,0.990)$ & 0.047 \\
\hline
\end{tabular}

Values are presented as odds ratio [95\% Cl (confidence interval)]. OR, odds ratio; PEEK, poly-ether-ether-ketone.

$\mathrm{P}<0.001)$ were found to be significantly associated with CR occurrence (Table 3, Figures 1,2).

\section{Discussion}

In the present study, the incidence of CR in older patients with TLIF was $1.74 \%$, which is consistent with that reported previously $(5,6,9,11)$. The multivariate analysis verified that screw loosening and endplate injury were significantly associated with CR.

CR is considered to be an early onset complication after surgery $(5,7,12)$. Kimura et al. (5) reported 9 cases of CR among 1,070 patients with posterior fixation, all of whom developed CR within 2 months postoperatively. In the present study, the majority of the CR cases occurred around 3 months after surgery. Therefore, we suggested that patients were followed up at 3 months after the operation. Lee et al. (6) reported that insufficient posterior fixation led to residual inter-segmental instability, which could result in posterior screw loosening and CR. We found that segmental instability due to the failure of earlier screw fixation resulted in CR. Previous studies have also reported inappropriate posterior screw fixation to be a major risk factor for CR $(13,14)$, and this theory is similarly applicable to CR patients who undergo posterior lumbar interbody fusion (15). Other reported risk factors for CR include total facetectomy, inappropriate posterior screw fixation, posterior hardware removal, unilateral pedicle screw fixation, fusion level involved L5/S1, multilevel fusion surgery, cage type and disc space, and osteoporosis $(5,13,16)$. The discrepancy between the findings of previous studies and those of the current investigation may have resulted from other authors paying more attention to the effect of surgical procedures and fixation patterns on the occurrence of CR. However, the influence of the surgeon's manipulation has largely been ignored, for example, screw loosening resulting from impertinent screw placement in osteoporotic vertebrae and endplate injury during inadvertent discectomy.

The current study failed to uncover a significant association between osteoporosis and CR, which may be due to many of the included osteoporotic patients receiving anti-osteoporosis treatment after admission, and the resulting improvement in BMD might have affected the incidence of CR. Wittenberg concluded that the risk of early loosening of pedicle screws was increased with a BMD of $\leq 90 \mathrm{mg} / \mathrm{mL}$ but decreased with a BMD of $\geq 120 \mathrm{mg} / \mathrm{mL}$ (17). Soshi et al. pointed out there were positive correlations between the screw pull-out force and BMD: when bone cement was used in an osteoporotic vertebral body, a two-fold stronger pull-out force was developed (18). Mo et al. compared cement-augmented pedicle screws with traditional pedicle screws for the treatment of osteoporotic lumbar degenerative disorders, and found that the former showed better fusion and had a lower screw-loosening rate, especially in patients with multi-level fusion (19).

Another contributing factor to segmental stability for cage fusion is the stability of the interface between the cage and vertebral endplate; however, this has been seldomly studied. Studies have highlighted the importance of keeping the bone endplate intact to prevent the cage subsidence or posterior migration (12,19-22). Park pointed out that there was not enough strength to support cage stabilization if endplate injury occurred during TLIF (9). We summarized two indicators of potential endplate injury: (I) the sudden loss of tension when the narrow disc space is distracted, indicating the push-through of the distractor into the endplate defect; (II) cortical or cancellous bone chips detected from the contents following endplate curetting. Rather than continue on the same side, the optimal alternative is to shift to the contralateral side for cage insertion. There are another two approaches for preventing this issue. One is to avoid using the distractor 

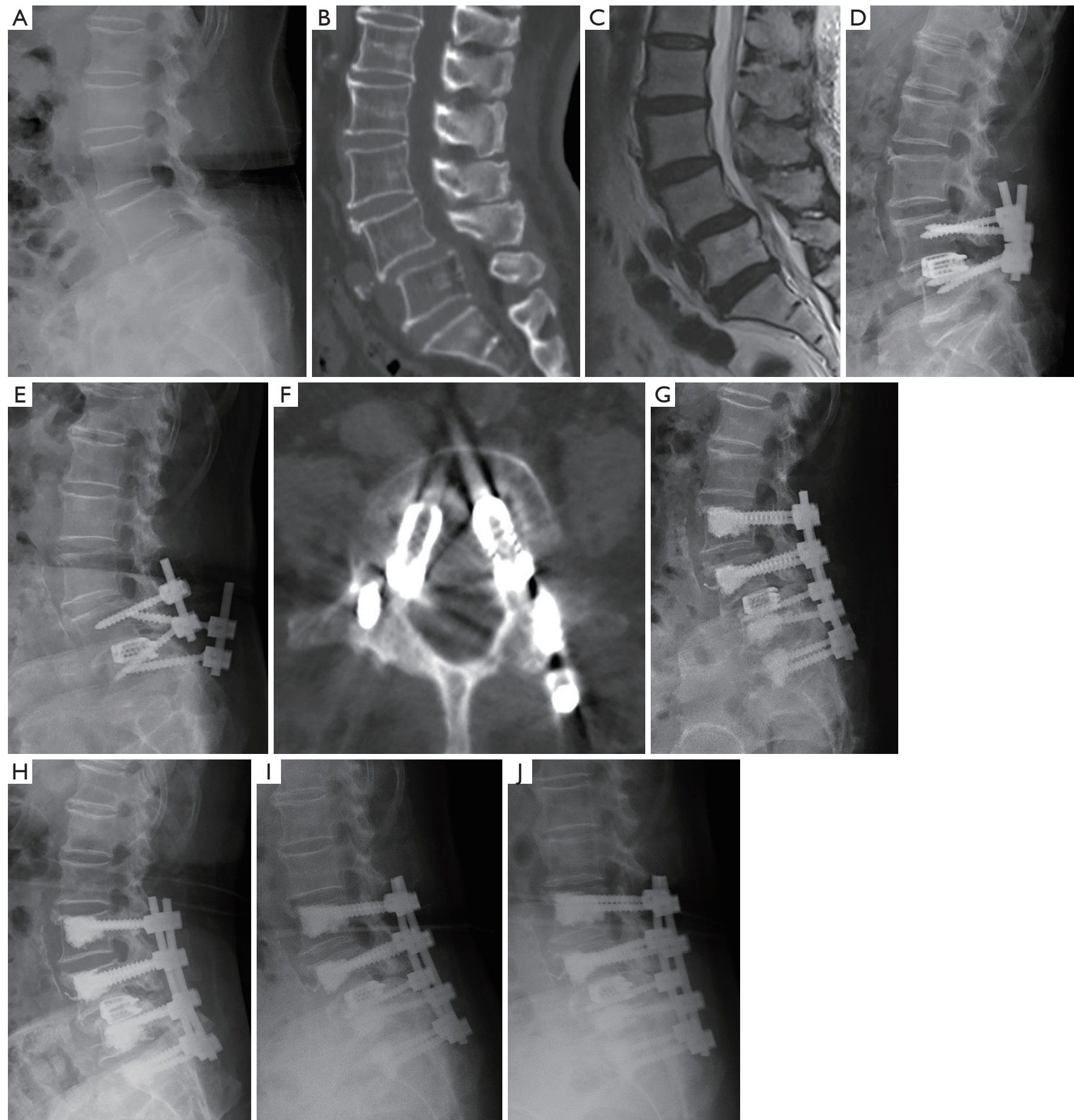

Figure 1 A 71-year-old female was diagnosed with degenerative spondylolisthesis and underwent L4/5 transforaminal lumbar interbody fusion (TLIF). (A,B,C) Pre-operative radiological examination; (D) lateral radiograph 4 days postoperatively; (E) cage retropulsion (CR) at L4/5 and screw loosening 3 months after surgery; (F) computed tomography images showed the posterior edges of both cages were behind the posterior edge of the vertebral body; $(G)$ lateral radiograph 7 days after revision surgery. More pedicle screws were inserted using the cement-augmentation technique, and the retropulsed cages were not revised, as the patient's chief complaint was lower back pain rather than radiculopathy; $(\mathrm{H}, \mathrm{I}, \mathrm{J})$ lateral radiographs obtained at 1, 2, and 3 years after revision surgery, respectively. The position of cages was maintained and no screw pull-out occurred. 

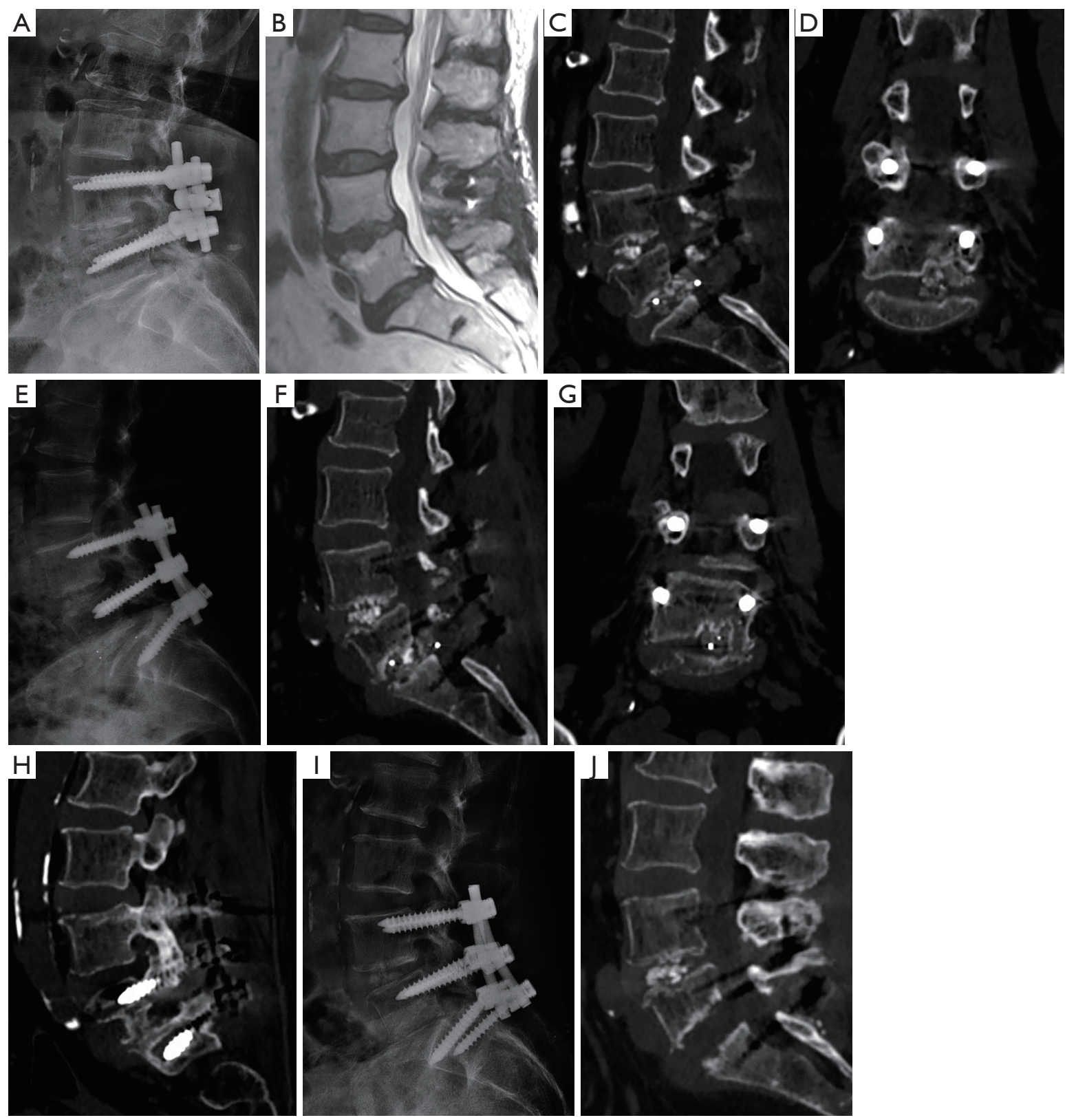

Figure 2 A 73 -year-old female underwent L4/5 transforaminal lumbar interbody fusion (TLIF) before admission. The patient was diagnosed with degenerative disc disease at L5/S1 and underwent in situ TLIF and L4-S1 bilateral posterior instrumentation. She reported sudden severe tingling along the left lower extremity on the 4th day postoperatively. Computed tomography (CT) did not show hematoma, but cage $(13 \mathrm{~mm} \times 27 \mathrm{~mm})$ retropulsion and endplate injury were detected. The revision surgery included repositioning by punching the cage into the disc space and effective compression to the disc was achieved through forcefully compressing the pedicle screws along the rods again. However, the patient's radiculopathy recurred and CT revealed screw loosening and cage retropulsion (CR) recurrence after 3 months. During the second revision, the retropulsed cage had to be removed, and the loosened left S1 screw was revised. At last, the definite bone resorption was verified from the CT 1 year postoperatively. (A,B) Preoperative radiological examination; (C,D) CT showed CR and that part of cage had breached into the cortical endplate; (E) lateral radiograph taken 4 days after the first revision. The cage was punched into the L5/S1 disc; (F,G,H) CR recurrence and loosening of the left S1 pedicle screw were revealed by CT at 3 months after the revision; (I) lateral radiograph taken 3 days after the second revision. The cage had to be removed and the loosened left S1 pedicle screw was revised; (J) CT image acquired 1 year after the first revision showing the clear bone resorption of the L5 lower endplate. 
to open the disc space. The other is to adjust the endplate preparation. The anterior two-thirds of the endplate can be prepared with the curette gently, the posterior third of the cartilaginous endplate should be polished gently. Therefore, great attention should be paid on the preparation for the endplate, especially its posterior third portion.

Revision surgery is sometimes inevitable for CR following lumbar fusion $(23,24)$. Technical modification for revision surgery should be advocated to prevent postoperative adverse events. Park recommended the following techniques to prevent cage migration (9): (I) the surgeon should check if the patient has abnormal BMD or pear-shaped disc before performing TLIF; (II) the cage should be inserted into the anterior portion of the intervertebral space and decrease endplate injury; (III) the use of double cages is preferable. Also, we have several clinical suggestions on technical modifications for preventing CR: (I) BMD evaluations should be routinely conducted for TLIF in older patients, and the pedicle screw designed for cement injection should be used in surgery; (II) a distractor should not be used to open disc space, as it may increase endplate injury; and (III) when endplate injury occurs on one side of the intervertebral space, the cage should be placed on the contralateral side. Generally, we suggested patients to perform follow-up at 1, 3, 6, 12 months after surgery. If mild CR was verified by imaging and patient was asymptomatic, we advocated wait-andsee strategy. If severe CR was found due to instrumental malposition like screw loosening, and the patient had corresponding symptoms, we would perform a revision surgery for the patient. Besides, in combination with the status of intervertebral fusion is important. If already fused and nerve compression by cage had occurred, resection of the posterior part of cage or removing the cage was need. If intervertebral fusion had not developed over 6 months and CR was occurrent, it depended on nerve compression. If had nerve compression, we performed revision surgery immediately, the cage would be removed and replaced with a bigger one through the original incision or another lateral approach. If CR was mild and had not nerve compression, we took wait-and-see strategy as well.

This study has some limitations. First, as mentioned above, the osteoporotic patients in our study had received anti-osteoporotic treatments before surgery, which was a confounding factor for determining if a correlation existed between osteoporosis and CR in older patients undergoing TLIF. Second, this retrospective study did not include a control group and different pedicle screws were used in the operations, both of which decreased the accuracy of the data. Third, it was difficult to analyze all the risk factors for CR; therefore, some potential risk factors associated with CR may have been omitted. Hence, more potential risk factors should be included and analyzed for CR in older patients with TLIF.

\section{Conclusions}

In this study, the incidence of CR in older patients who underwent TLIF was $1.74 \%$. Screw loosening and endplate injury were found to be independently associated with CR. Intraoperatively, bilateral fixation with screws and rods are advocated to avoid screw loosening. Spine surgeons should improve technical manipulation to decrease endplate injury.

\section{Acknowledgments}

Funding: None.

\section{Footnote}

Reporting Checklist: The authors have completed the STROBE reporting checklist. Available at http://dx.doi. org/10.21037/atm-20-7416

Data Sharing Statement: Available at http://dx.doi. org/10.21037/atm-20-7416

Conflicts of Interest: All authors have completed the ICMJE uniform disclosure form (available at http://dx.doi. org/10.21037/atm-20-7416). The authors have no conflicts of interest to declare.

Ethical Statement: The authors are accountable for all aspects of the work in ensuring that questions related to the accuracy or integrity of any part of the work are appropriately investigated and resolved. This retrospective study was approved by the Institutional Review Board of Beijing Jishuitan Hospital (202007-24). All procedures performed in this study involving human participants were in accordance with the Declaration of Helsinki (as revised in 2013). Individual consent for this retrospective analysis was waived.

Open Access Statement: This is an Open Access article distributed in accordance with the Creative Commons Attribution-NonCommercial-NoDerivs 4.0 International 
License (CC BY-NC-ND 4.0), which permits the noncommercial replication and distribution of the article with the strict proviso that no changes or edits are made and the original work is properly cited (including links to both the formal publication through the relevant DOI and the license). See: https://creativecommons.org/licenses/by-nc-nd/4.0/.

\section{References}

1. Harms J, Rolinger H. A one-stager procedure in operative treatment of spondylolistheses: dorsal traction-reposition and anterior fusion (author's transl). Z Orthop Ihre Grenzgeb 1982;120:343-7.

2. Taneichi H, Suda K, Kajino T, et al. Unilateral transforaminal lumbar interbody fusion and bilateral anterior-column fixation with two Brantigan I/F cages per level: clinical outcomes during a minimum 2-year followup period. J Neurosurg Spine 2006;4:198-205.

3. Aygün H, Cakar A, Huseyinoglu N, et al. Clinical and radiological comparison of posterolateral fusion and posterior interbody fusion techniques for multilevel lumbar spinal stabilization in manual workers. Asian Spine J 2014;8:571-80.

4. Pawar AY, Hughes AP, Sama AA, et al. A comparative study of lateral lumbar interbody fusion and posterior lumbar interbody fusion in degenerative lumbar spondylolisthesis. Asian Spine J 2015;9:668-74.

5. Kimura H, Shikata J, Odate S, et al. Risk factors for cage retropulsion after posterior lumbar interbody fusion: analysis of 1070 cases. Spine 2012;37:1164-9.

6. Lee DY, Park YJ, Song SY, et al. Risk Factors for Posterior Cage Migration after Lumbar Interbody Fusion Surgery. Asian Spine J 2018;12:59-68.

7. Pan FM, Wang SJ, Yong ZY, et al. Risk factors for cage retropulsion after lumbar interbody fusion surgery: Series of cases and literature review. Int J Surg 2016;30:56-62.

8. Uzi EA, Dabby D, Tolessa E, et al. Early retropulsion of titanium-threaded cages after posterior lumbar interbody fusion. a report of two cases. Spine 2001;26:1073-5.

9. Park MK, Kim KT, Bang WS, et al. Risk factors for cage migration and cage retropulsion following transforaminal lumbar interbody fusion. Spine J 2019;19:437-47.

10. Yuan Q, Zhang G, Wu J, et al. Clinical evaluation of the polymethylmethacrylate-augmented thoracic and lumbar pedicle screw fixation guided by the three-dimensional navigation for the osteoporosis patients. Eur Spine J 2015;24:1043-50.

11. Zhao FD, Yang W, Shan Z, et al. Cage migration after transforaminal lumbar interbody fusion and factors related to it. Orthop Surg 2012;4:227-32.

12. Aoki Y, Yamagata M, Nakajima F, et al. Examining risk factors for posterior migration of fusion cages following transforaminal lumbar interbody fusion: a possible limitation of unilateral pedicle screw fixation. J Neurosurg Spine 2010;13:381-7.

13. Corniola MV, Jaersberg M, Stienen MN, et al. Complete cage migration/subsidence into the adjacent vertebral body after posterior lumbar interbody fusion. J Clin Neurosci 2015;22:597-8.

14. Chen L, Yang H, Tang T. Cage migration in spondylolisthesis treated with posterior lumbar interbody fusion using BAK cages. Spine 2005;30:2171-5.

15. Lund T, Oxland TR, Jost B, et al. Interbody cage stabilisation in the lumbar Spine-biomechanical evaluation of cage design, posterior instrumentation and bone density. J Bone Joint Surg Br 1998;80:351-9.

16. Abbushi A, Cabraja M, Thomale UW, et al. The influence of cage positioning and cage type on cage migration and fusion rates in patients with monosegmental posterior lumbar interbody fusion and posterior fixation. Eur Spine J 2009;18:1621-8.

17. Wittenberg RH, Shea MS, Swartz DE, et al. Importance of bone mineral density instrumental spine fusion. Spine 1991;16:647-52.

18. Soshi S, Shiba R, Kondo H, et al. An experimental study on transpedicular screw fixation in relation to osteoporosis of the lumbar spine. Spine 1991;16:1335-41.

19. Mo GY, Guo HZ, Guo DQ, et al. Augmented pedicle trajectory applied on the osteoporotic spine with lumbar degenerative disease: mid-term outcome. J Orthop Surg Res 2019;14:170.

20. Abbushi A, Cabraja M, Thomale UW, et al. The influence of cage positioning and cage type on cage migration and fusion rates in patients with monosegmental posterior lumbar interbody fusion and posterior fixation. Eur Spine J 2009;18:1621-8.

21. Kozak JA, Heilman AE, O'Brien JP. Anterior lumbar fusion options. Technique and graft materials. Clin Orthop Relat Res 1994;300:45-51.

22. Kuslich SD, Ulstrom CL, Griffith SL, et al. The Bagby and Kuslich method of lumbar interbody fusion. History, techniques, and 2-year follow-up results of a United States prospective, multicenter trial. Spine 1998;23:1267-78.

23. Hu YH, Niu CC, Heieh MK, et al. Cage positioning as a risk factor for posterior cage migration following transforaminal lumbar interbody fusion -an analysis of 953 
cases. BMC Musculoskelet Disord 2019;20:260.

24. Lee JG, Lee SM, Kim SW, et al. Repeated migration of a fusion cage after posterior lumbar interbody fusion.

Cite this article as: Li N, Dai M, Zhang B, He D, Wei Y, Duan F, Sun Y, Liu B, Mo F, Tian W. Risk factors for cage retropulsion after transforaminal lumbar interbody fusion in older patients. Ann Transl Med 2020;8(24):1660. doi: 10.21037/ atm-20-7416
Korean J Spine 2013;10:25-7.

(English Language Editor: J. Reynolds) 